\title{
Molecular breeding of Saccharomyces cerevisiae with high RNA content by harnessing essential ribosomal RNA transcription regulator
}

\author{
Yu Sasano ${ }^{1}$, Takahiro Kariya', Shogo Usugi ${ }^{1}$, Minetaka Sugiyama ${ }^{1}$ and Satoshi Harashima2 ${ }^{*}$
}

\begin{abstract}
As yeast is commonly used for RNA production, it is industrially important to breed strains with high RNA contents. The upstream activating factor (UAF) plays an important role in transcription of ribosomal RNA (rRNA), a major constituent of intracellular RNA species. Here, we targeted the essential rRNA transcription regulator Rrn5 of Saccharomyces cerevisiae, a component of the UAF complex, and disrupted the genomic RRN5 gene using a helper plasmid carrying an RRN5 gene. Then we isolated nine suppressor mutants (Sup mutants) of RRN5 gene disruption, causing deficiency in rRNA transcription. The Sup mutants had RNA contents of approximately $40 \%$ of the wild type level and expansion of rDNA repeats to ca. 400-700 copies. Reintroduction of a functional RRN5 gene into Sup mutants caused a reduction in the number of rDNA repeats to close to the wild type level but did not change RNA content. However, we found that reintroduction of RRN5 into the Sup16 mutant (in which the FOB1 gene encoding the rDNA replication fork barrier site binding protein was disrupted) resulted in a significant increase (17\%) in RNA content compared with wild type, although the rDNA repeat copy number was almost identical to the wild type strain. In this case, upregulated transcription of non-transcribed spacers (NTS) occurred, especially in the NTS2 region; this was likely mediated by RNA polymerase II and accounted for the increased RNA content. Thus, we propose a novel breeding strategy for developing high RNA content yeast by harnessing the essential rRNA transcription regulator.
\end{abstract}

Keywords: S. cerevisiae, RNA content, Non-transcribed spacer (NTS), rDNA, Rrn5, Fob1

\section{Introduction}

Ribonucleic acids (RNA) and nucleotides such as $5^{\prime}$-IMP and $5^{\prime}$-GMP have numerous useful properties. Many researches have shown the efficacy of RNA intake e.g. promotion of tissue differentiation (Rathbone et al. 1992), and immunostimulatory activity (Barbalat et al. 2011). $5{ }^{\prime}$-IMP and 5'-GMP are produced by breaking down of yeast RNA and subsequent conversion of 5'-AMP to 5'-IMP by AMP deaminase and they are well known for "umami" taste and used as food additives (Kurihara and Kashiwayanagi 2000). A budding yeast Saccharomyces cerevisiae is the most common microorganism for producing RNA because of its high RNA content (Warner

\footnotetext{
*Correspondence: harashima@bio.sojo-u.ac.jp

${ }^{2}$ Department of Applied Microbial Technology, Faculty of Biotechnology

and Life Science, Sojo University, Ikeda 4-22-1, Kumamoto-shi,

Kumamoto 860-0082, Japan

Full list of author information is available at the end of the article
}

1999). Because ribosomal RNA (rRNA) accounts for approximately $80 \%$ of total RNA in yeast, elevating intracellular rRNA level is the key to construct a yeast strain with high RNA content.

In $S$. cerevisiae, rDNA encoding rRNA are tandemly repeated on chromosome XII and rDNA repeats consists of 100-150 copies (Petes 1979). Each unit is composed of two transcribed regions, $35 \mathrm{~S}$ precursor rRNA and $5 \mathrm{~S}$ rRNA, and two intergenic non-transcribed spacers, NTS1 and NTS2 (Fig. 1). The 35 S precursor rRNA is normally transcribed by RNA polymerase I ( $\mathrm{Pol} \mathrm{I}$ ) and the 5S rRNA is transcribed by RNA polymerase III ( $\mathrm{Pol} \mathrm{III}$ ). Transcriptional regulation of 35S rRNA gene is exerted by four factors: core factor (CF), Rrn3, TATA box binding protein (TBP), and upstream activating factor (UAF). $\mathrm{CF}$ and Rrn3 are indispensable for basal level transcription of 35S rRNA (Keys et al. 1994; Lalo et al. 1996; Lin et al. 1996; Yamamoto et al. 1996), whereas UAF and 


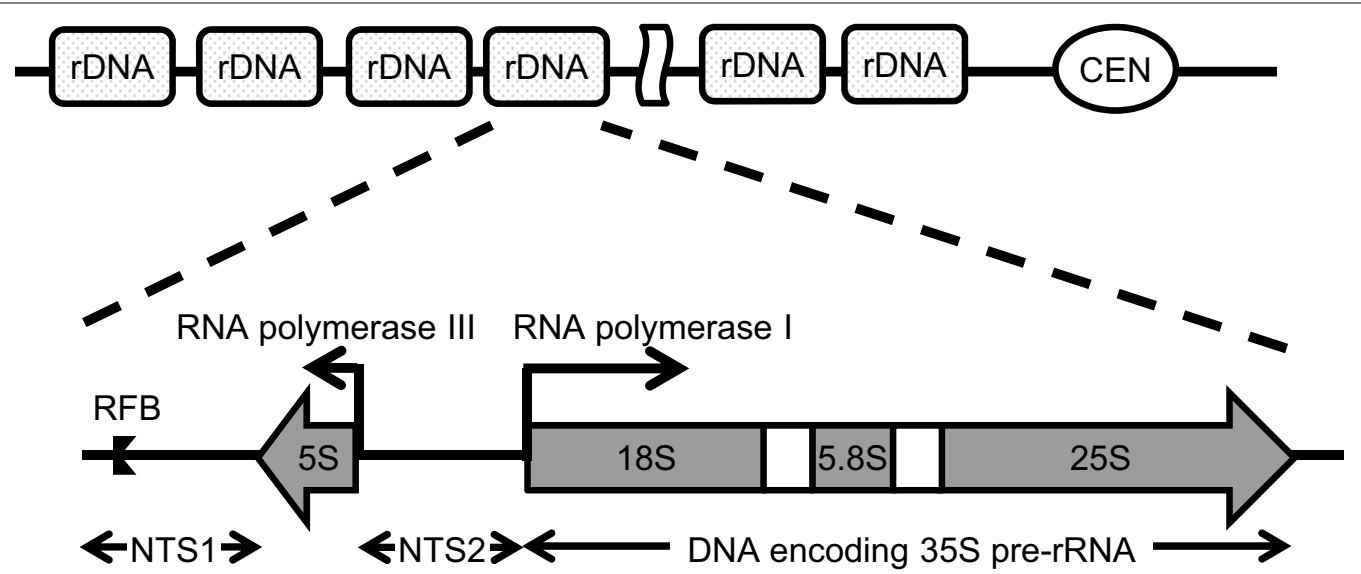

Fig. 1 Schematic of a rDNA unit in S. cerevisiae. rDNA cluster is located at chromosome XII in S. cerevisiae. rDNA repeats consists of 100-150 copies. Each rDNA unit contains two transcribed regions, 355 precursor rRNA and 5 S rRNA, and two intergenic non-transcribed spacers, NTS1 and NTS2. RFB, replication fork barrier site

TBP are required for high level transcription (Keener et al. 1998; Keys et al. 1996; Steffan et al. 1996). UAF is composed of Rrn5, Rrn9, Rrn10, histone H3, histone H4, and Uaf30 (Goetze et al. 2010). UAF binds to upstream element located at upstream of the $35 \mathrm{~S}$ pre-rRNA gene to activate the transcription of the $35 \mathrm{~S}$ precursor RNA gene by recruiting CF and Pol I. In the NTS1 region, there is a replication fork barrier site (RFB), to which fork block protein (Fob1) binds. RFB and Fob1 protein play an important role in maintenance of rDNA repeat copy number at proper level through regulation of rDNA recombination (Kobayashi 2011). It is known that deletion of $F O B 1$ gene suppresses rDNA recombination and thereby rDNA repeat copy number becomes invariable (Defossez et al. 1999; Johzuka and Horiuchi 2002; Kobayashi et al. 1998).

Fob1 and UAF play an important role in silencing of RNA polymerase II (Pol II)-dependent transcription at 35S rRNA and NTS regions. In UAF-defective mutant caused by disruption of essential component $\operatorname{Rrn} 5$ or Rrn9, drastic chromatin remodeling is induced allowing access of Pol II to 35S rRNA promoter, and consequently Pol II-dependent transcription of 35S rRNA, a phenomenon called polymerase switch (PSW) occurs (Goetze et al. 2010; Vu et al. 1999). Furthermore, copy number of rDNA repeat is increased by several fold (Oakes et al. 1999).

Previously, we focused on Rrn10, one of the UAF component and isolated suppressor mutant of rrn10 disruption. We succeeded in breeding of strains with increased RNA content compared with wild type by reintroduction of functional RRN10 gene into the suppressor mutants (Chuwattanakul et al. 2011, 2012; Khatun et al. 2013a, b). Because RRN10 is a non-essential gene, this success prompted us to isolate suppressor mutants of disruption of genes encoding essential UAF components, such as RRN5. This was based on the idea that suppressor mutation of disruption of essential genes would have stronger effect than that of non-essential genes in terms of restoration of rDNA transcription activity. Thus, in this study, we isolated suppressor mutants of $r$ rn5 disruption and by utilizing these mutants we constructed a yeast strain exhibiting high RNA content probably due to increased transcription of NTS region which is normally silenced.

\section{Materials and methods}

\section{Strains and media}

Saccharomyces cerevisiae strain SH6471 (NBRP-Yeast, Japan) was used as a parental strain in this study. The detailed information about strains used in this study is shown in Table 1. Yeast cells were grown in YPDA medium consisting of 5\% YPD broth (Sigma Aldrich, St. Louis, MO, USA) and $0.04 \%$ adenine (Wako, Osaka, Japan) or in SC medium consisting of $0.67 \%$ yeast nitrogen base without amino acids (Becton, Dickinson and Company, Franklin Lakes, NJ, USA), 0.2\% drop out mix, and $2 \%$ glucose. SC medium lacking particular amino acids was used for auxotrophic marker selection. For solid media, 2\% agar was used to solidify the medium. Escherichia coli DH5 $\alpha$ was used for plasmid construction and propagation; E. coli recombinant strains were grown in Luria-Bertani (LB) medium containing $100 \mu \mathrm{g} / \mathrm{mL}$ ampicillin.

\section{Disruption of the RRN5 gene}

Because the RRN5 gene is an essential gene, we introduced a plasmid harboring RRN5 gene region marked with URA3 (pRRN5) into SH6471 strain before disruption 
Table 1 S. cerevisiae strains and plasmids used in this study

\begin{tabular}{|c|c|}
\hline Name & Description \\
\hline \multicolumn{2}{|l|}{ Strain } \\
\hline SH6471 & 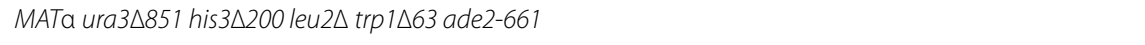 \\
\hline SH8836 & 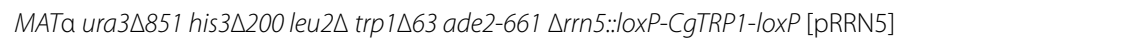 \\
\hline SH8894 & 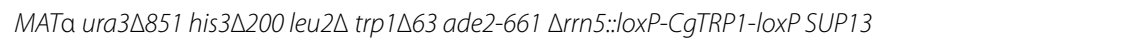 \\
\hline SH8895 & 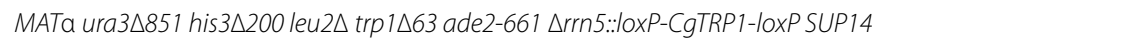 \\
\hline SH8896 & 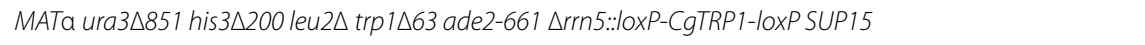 \\
\hline SH8897 & 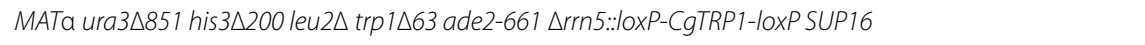 \\
\hline SH8898 & 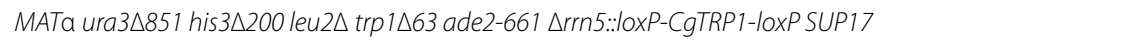 \\
\hline SH8899 & 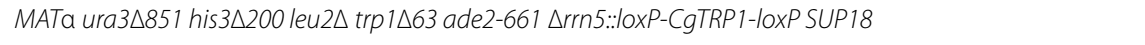 \\
\hline SH8900 & 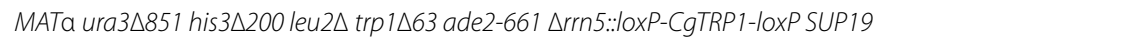 \\
\hline SH8904 & 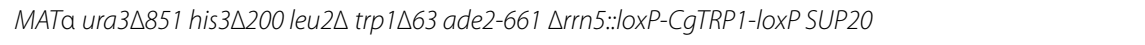 \\
\hline SH8905 & 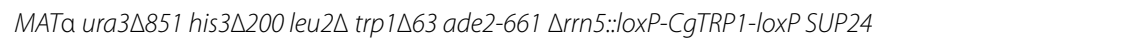 \\
\hline SH30025 & $\mathrm{Ura}^{+}$transformants of SH8894 with pRRN5 \\
\hline SH30026 & Ura $^{+}$transformants of SH8895 with pRRN5 \\
\hline SH30027 & Ura $^{+}$transformants of SH8896 with pRRN5 \\
\hline SH30028 & $\mathrm{Ura}^{+}$transformants of SH8897 with pRRN5 \\
\hline SH30029 & $\mathrm{Ura}^{+}$transformants of SH8898 with pRRN5 \\
\hline SH30030 & Ura $^{+}$transformants of SH8899 with pRRN5 \\
\hline SH30031 & $\mathrm{Ura}^{+}$transformants of SH8900 with pRRN5 \\
\hline SH30035 & $\mathrm{Ura}^{+}$transformants of SH8904 with pRRN5 \\
\hline SH30036 & Ura $^{+}$transformants of SH8905 with pRRN5 \\
\hline TK1 & 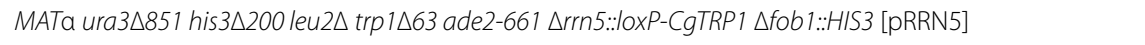 \\
\hline TK2 & $\triangle f \circ b 1:: H I S 3$ disruptant of SH8894 \\
\hline TK3 & $\Delta$ fob $1::$ HIS3 disruptant of SH8895 \\
\hline TK4 & $\triangle f \circ b 1:: H I S 3$ disruptant of SH8896 \\
\hline TK5 & $\Delta$ fob $1::$ HIS3 disruptant of SH8897 \\
\hline TK6 & $\triangle f \circ b 1:: H I S 3$ disruptant of SH8898 \\
\hline TK7 & $\Delta f \circ b 1:: H I S 3$ disruptant of SH8899 \\
\hline TK8 & $\triangle f \circ b 1:: H I S 3$ disruptant of SH8900 \\
\hline TK9 & $\triangle f o b 1:: H I S 3$ disruptant of SH8904 \\
\hline TK10 & $\Delta f \circ b 1:: H I S 3$ disruptant of SH8905 \\
\hline TK11 & $\mathrm{Ura}^{+}$transformants of TK2 with pRRN5 \\
\hline TK12 & $\mathrm{Ura}^{+}$transformants of TK5 with pRRN5 \\
\hline \multicolumn{2}{|l|}{ Plasmid } \\
\hline pRRN5 & YCp-URA3-RRN5 (A centromere type plasmid harboring functional RRN5 gene marked with URA3 gene) \\
\hline p2453 & pUC18 plasmid harboring FOB1 gene disruption cassette (a kind gift from T. Kobayashi) \\
\hline
\end{tabular}

of the RRN5 gene. The pRRN5 plasmid was constructed as follows. The RRN5 gene region including promoter, open reading frame, and terminator, was amplified by PCR using oligonucleotide primer pair, CDR-RRN5-F and CDR-RRN5-R, and genomic DNA of SH6471 strain as a template. The amplified fragment was ligated into the SmaI site of pRS316 plasmid (Sikorski and Hieter 1989). The resulting plasmid was named pRRN5. After introduction of pRRN5 into SH6471 strain, the RRN5 gene was disrupted as follows. The disruption cassette containing Candida glabrata TRP1 marker gene (CgTRP1) was prepared by PCR using oligonucleotide primer pair DR-RRN5-F and DR-RRN5-R, and p3010 plasmid (Sugiyama et al. 2005) as a template. The amplified fragment was introduced into SH6471 strain harboring pRRN5. The obtained transformants $\left(\operatorname{Trp}^{+}\right)$was checked for disruption of the genomic RRN5 gene by colony direct PCR using oligonucleotide primer pairs, DR-RRN5-F and DRRRN5-R, and, CDR-RRN5-F and CDR-RRN5-R (data not shown). The obtained $r r n 5$ disruptant was named SH8836. Sequence of the oligonucleotide primers used in this study is shown in Additional file 1: Table S1. 


\section{Screening of suppressor mutants}

SH8836 strain was cultivated on SC solid medium lacking uracil and tryptophan, and then cells were replicated onto SC solid medium lacking tryptophan and containing 5-fuloroorotic acid (5-FOA) and uracil for curing pRRN5 plasmid. Twenty-four suppressor mutants were isolated and then, absence of RRN5 gene was confirmed by PCR using oligonucleotide primer pair ORF-RRN5-F and ORF-RRN5-R (data not shown). Finally, nine suppressor mutants were obtained (Sup13, Sup14, Sup15, Sup16, Sup17, Sup18, Sup19, Sup23, and Sup24).

\section{Measurement of total RNA content}

Total intracellular RNA content in yeast was measured by the perchloric acid (PCA) method (Chuwattanakul et al. 2011) with some modifications. Yeast strains were cultivated at $30{ }^{\circ} \mathrm{C}$ in $\mathrm{SC}$ medium supplemented with appropriate nutrients. Cultured cells were inoculated in $5 \mathrm{~mL}$ of the same medium at initial $\mathrm{OD}_{660}=0.2$ and incubated until $\mathrm{OD}_{660}$ reached 1.0. The cell culture was divided into three tubes $\left(1 \mathrm{~mL}\right.$ each) and $\mathrm{OD}_{600}$ in each tube was measured. After centrifugation of the cell culture $(12,000 \mathrm{rpm}, 30 \mathrm{~s})$, the supernatant was removed and $1 \mathrm{~mL}$ of $0.5 \mathrm{~N}$ perchloric acid (Wako, Osaka, Japan) was added and resuspended. After incubation at $70{ }^{\circ} \mathrm{C}$ for $20 \mathrm{~min}$, cells were separated by centrifugation $(12,000 \mathrm{rpm}, 2 \mathrm{~min})$ and absorbance at $260 \mathrm{~nm}\left(\mathrm{~A}_{260}\right)$ of the supernatant was measured. We calculated total RNA content by using the following equation.

$$
\begin{aligned}
& \text { RNA content }(\mathrm{mg} / \mathrm{g} \text {-Dry cell weight }) \\
& =\frac{A_{260} \times 0.0368}{O D_{600} \times 0.257 \times 0.001}
\end{aligned}
$$

Dry cell weight was determined from the standard curve of $\mathrm{OD}_{600}$ vs. dry cell weight using SH6471 strain. The mean value and standard deviation were calculated from three divided aliquots of the cell culture.

\section{Pulsed field gel electrophoresis (PFGE) and Southern hybridization}

Chromosomal DNAs from S. cerevisiae cultured in SC medium supplemented with appropriate nutrients were embedded in agarose plugs as described by Sheehan and Weiss (Sheehan and Weiss 1990). Chromosomes were separated by CHEF-DR ${ }^{\circledR}$ III pulsed field gel electrophoresis system (Bio-Rad, Hercules, $\mathrm{Ca}$, USA) on $0.8 \%$ gel in $1 \times$ TAE (0.04 M Tris base, $0.03 \mathrm{M}$ acetic acid, and $1 \mathrm{mM}$ EDTA) buffer at $14{ }^{\circ} \mathrm{C}$. The electrophoretic condition is as follows: voltage, $3 \mathrm{~V} / \mathrm{cm}$; switch time, $500 \mathrm{~s}$; angle, $106^{\circ}$; total running time, $48 \mathrm{~h}$. After staining with ethidium bromide, DNA was transferred onto Hybond ${ }^{\mathrm{TM}}-\mathrm{N}^{+}$membrane (GE Healthcare, Chicago, IL, USA) by capillary blotting. Probe labelling, hybridization, and signal detection were carried out by ECL Direct ${ }^{\mathrm{TM}}$ nucleic acid labeling and detection system (GE Healthcare, Chicago, IL, USA). The probe used for detection of rDNA repeat was prepared by PCR amplifying a part of the 5S rRNA gene using oligonucleotide primers SB-probe_5S-F and SB-probe_5S-R, and genomic DNA of SH6471 as a template.

\section{Quantitative real-time PCR}

For determination of rDNA repeat copy number, genomic DNA was extracted by Dr. GenTLE ${ }^{\circledR}$ (from yeast) High Recovery Kit (Takara Bio Inc., Shiga, Japan). Oligonucleotide primers RT-PCR_18S-F and RT-PCR_18S-R were used for amplification of $18 \mathrm{~S}$ rRNA gene and RTPCR_ACT1-F and RT-PCR_ACT1-R were for that of $A C T 1$ gene. For RNA isolation, yeast cells cultivated on SC medium supplemented with appropriate nutrients were collected when $\mathrm{OD}_{600}$ reached 1.0. Then, RNA was extracted by RNeasy mini kit (Qiagen, Hilden, Germany). Reverse transcription was performed using QuantiTect Reverse Transcription Kit (Qiagen, Hilden, Germany). Quantitative real-time PCR was performed using SYBR ${ }^{\circledR}$ Premix Ex Taq ${ }^{\mathrm{TM}}$ II (Tli RNaseH Plus) (Takara Bio Inc., Shiga, Japan) and Thermal Cycler Dice ${ }^{\circledR}$ Real Time System (Takara Bio Inc., Shiga, Japan). Oligonucleotide primers used for quantitative real-time PCR are listed in Additional file 1: Table S1. Transcription level of ACT1 gene was used as an internal control.

\section{Disruption of the $F O B 1$ gene}

The disruption of the FOB1 gene was performed using p2453 plasmid (Kobayashi and Horiuchi 1996). The $3.4 \mathrm{~kb}$ EcoRI fragment of p2453 was introduced into SH8836 strain or Sup mutants. Transformants (His ${ }^{+}$) were further subjected to colony direct PCR to check whether the $F O B 1$ gene was disrupted using an oligonucleotide primer pair FOB1_deletion check-F and FOB1_ deletion check-R.

\section{Results}

Screening of suppressor mutants of rrn5 disruption

In our previous research, we focused on RRN10 gene, which is one of the components of UAF, and isolated suppressor mutants of growth defect of rrn10 disruption. By reintroduction of functional RRN10 gene into the suppressor mutants, we succeeded in constructing a mutant exhibiting higher RNA content than wild type (Chuwattanakul et al. 2011). This success gave us the idea that isolation and utilization of suppressor mutants of gene disruptant encoding essential UAF components such as Rrn5 would lead to construction of mutants with much higher RNA content. Therefore, we tried to isolate suppressor mutants of $r r n 5$ disruption. Because RRN5 gene is an essential gene, a helper plasmid pRRN5 harboring 

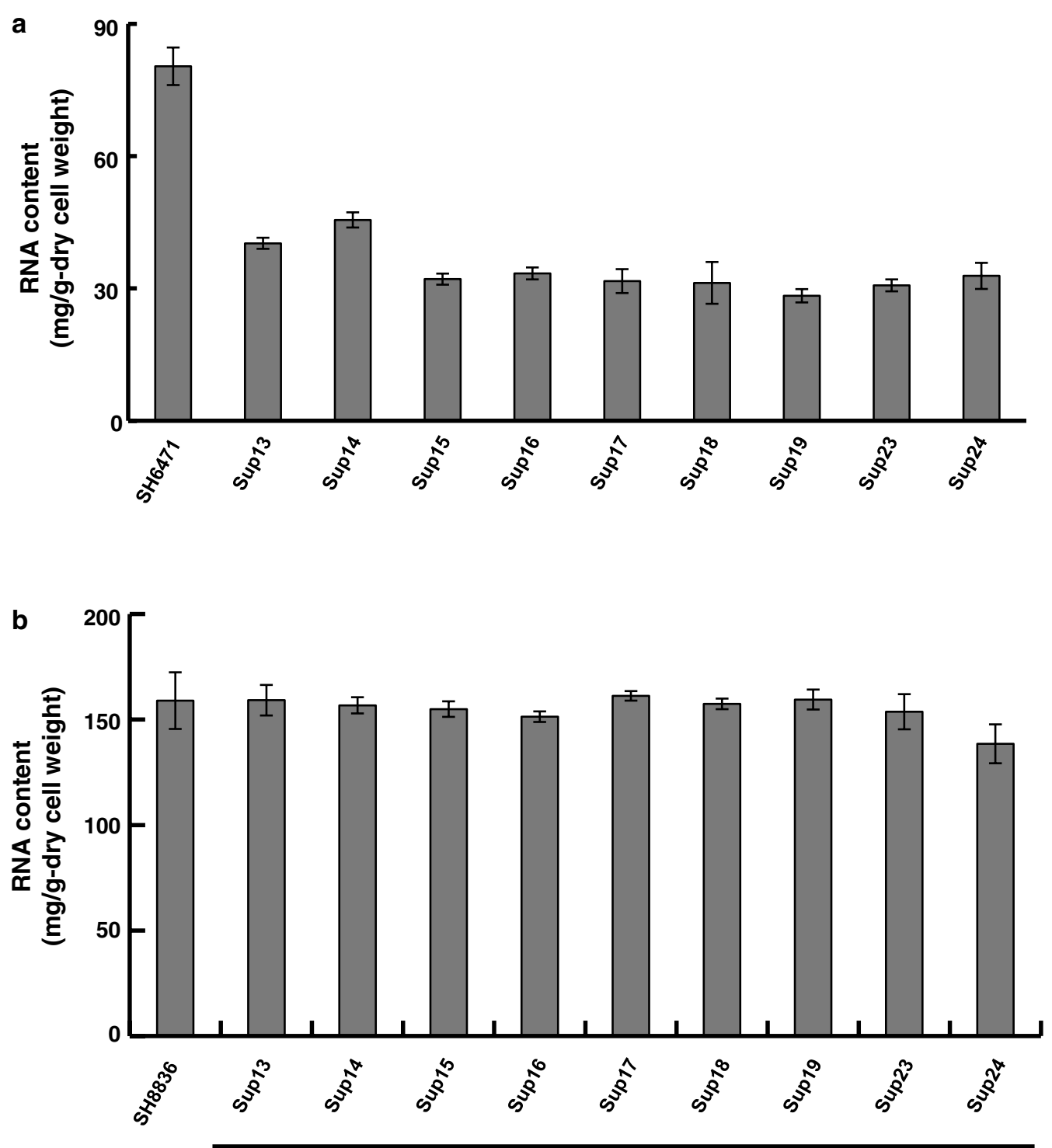

+ [pRRN5]

Fig. 2 Intracellular total RNA content of Sup mutants. a Intracellular total RNA content of nine Sup mutants normalized by dry cell weight. Cells were cultivated in YPDA liquid medium and harvested at middle exponential phase. Intracellular RNA content was measured by PCA method. b Intracellular total RNA content of nine Sup mutants harboring pRRN5 plasmid. Cells were cultivated in YPDA liquid medium and harvested at middle exponential phase. Intracellular RNA content was measured by PCA method

wild type RRN5 gene expression cassette marked with $U R A 3$ gene was introduced into SH6471 strain prior to RRN5 gene disruption. Then, genomic RRN5 gene was disrupted and the obtained disruptant was cultivated on 5-FOA medium in order to remove pRRN5 plasmid. Strains that can grow on 5-FOA medium were expected to have suppressor mutations of rrn5 disruption. From this screening, we isolated 24 suppressor mutants that could grow on 5-FOA medium. After confirmation of the absence of RRN5 gene by colony direct PCR, we finally obtained 9 suppressor mutants (Sup mutants) of rrn5 gene disruption. They were named strains Sup13, Sup14, Sup15, Sup16, Sup17, Sup18, Sup19, Sup21 and Sup24, respectively.

\section{Characterization of Sup mutants}

We performed dominant/recessive test by crossing Sup mutants and $\Delta r r n 5$ strain with a helper plasmid. This demonstrated that Sup13, Sup14, Sup16, and Sup24 have dominant mutation(s) and Sup15, Sup17, Sup18, Sup19, 
and Sup23 have recessive mutation(s). (data not shown). In addition, tetrad analysis of the diploids constructed by crossing Sup mutants and $\Delta r r n 5$ strain with a helper plasmid revealed that only Sup 15 and Sup16 have a single suppressor gene, whereas the other suppressor mutants have multiple suppressor genes (data not shown). All suppressor mutants could grow on YPDA liquid medium without helper plasmid, although the growth were severely retarded compared with SH8836 strain (Additional file 1: Figure S1). Relative growth rate of suppressor mutants was approximately $10 \%$ of the control strain (Additional file 1: Table S2). Total RNA content of the suppressor mutants at middle exponential phase was approximately $40 \%$ of the control strain (Fig. 2a). We introduced pRRN5 plasmid into suppressor mutants expecting that transformants will show increased RNA content compared with wild type strain as in the case of suppressor mutants of rrn10 disruptant (Chuwattanakul et al. 2011). Unexpectedly, total RNA content of all the suppressor mutants having pRRN5 plasmid was almost the same as SH8836 strain (Fig. 2b). This result suggested that the suppression mechanism of rrn5 disruption was dependent on UAFdeficiency and different from that of rrn10 disruption.
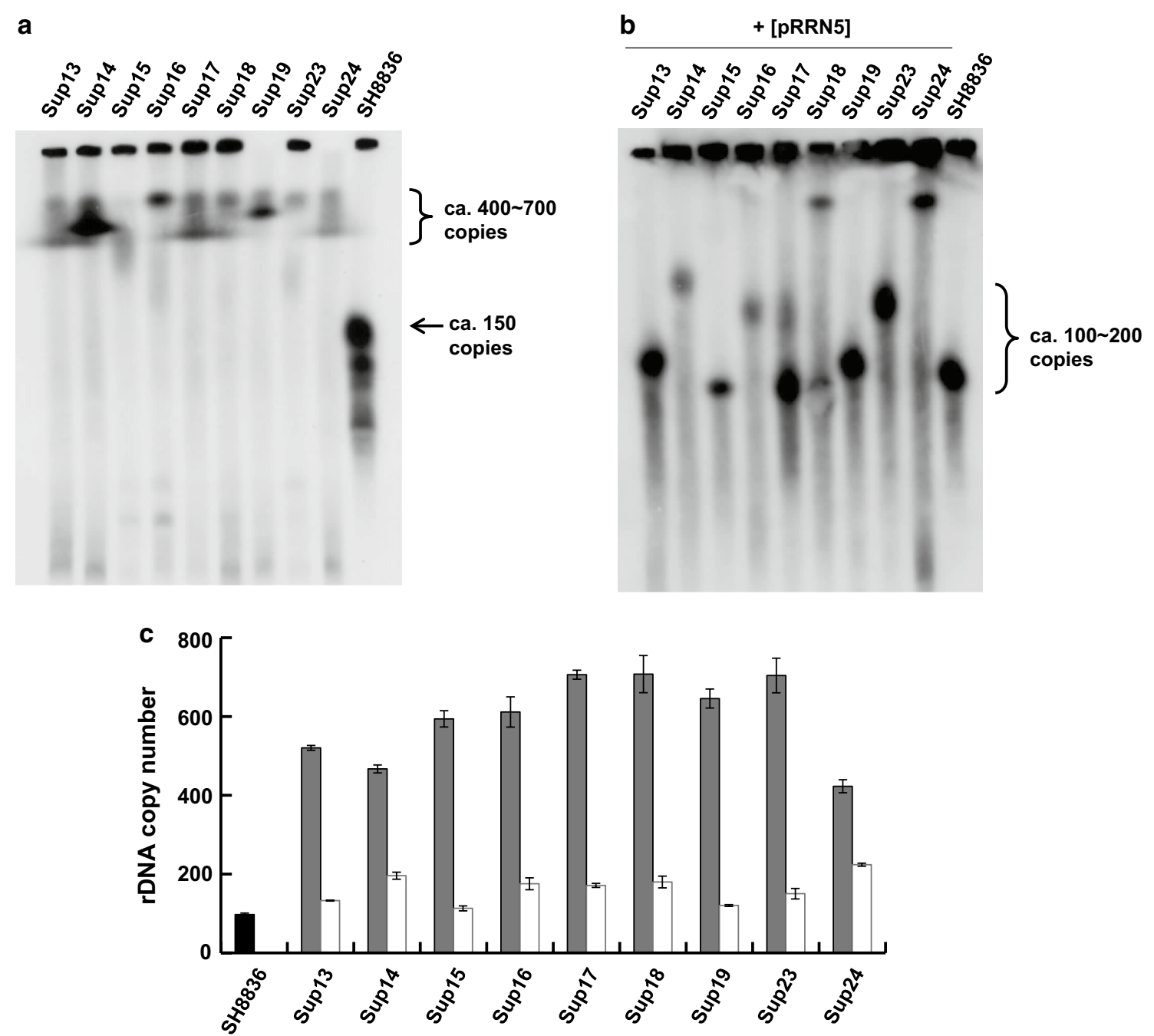

Fig. 3 Determination of rDNA copy number of Sup mutants. a Sup mutants were subjected to Southern blotting using 5 S rRNA gene as a probe after pulsed field gel electrophoresis. The curly bracket indicates expanded rDNA repeats in Sup mutants. Broad bands indicate that cell cultures are heterogeneous state. The arrow indicates rDNA repeat in SH8836 control strain. b Sup mutants harboring pRRN5 were subjected to Southern blotting using $5 \mathrm{~S}$ rRNA gene as a probe after pulsed field gel electrophoresis. $\mathbf{c}$ Precise determination of rDNA copy number in Sup mutants by quantitative real-time PCR. A primer pair RT-PCR_18S-F and RT-PCR_18S-R were used for detection of rDNA unit. ACT1 gene was used as the standard for calculation of rDNA copy number. Grey bars Sup mutants, white bars Sup mutants harboring pRRN5. The sequence of oligonucleotide primers used in this experiment is listed in Additional file 1: Table S1 
Previously, Oakes et al. reported that in UAF-deficient mutant, a switching of 35S rRNA transcription from by RNA polymerase I to by RNA polymerase II (called polymerase switch) and concomitant rDNA repeat expansion occur (Oakes et al. 1999; Vu et al. 1999). Polymerase switch and rDNA expansion is reversible; reintroduction of functional UAF lead to turning back to Pol I-dependent $35 \mathrm{~S}$ rRNA transcription and rDNA repeat contraction (Oakes et al. 1999; Vu et al. 1999). In order to measure the copy number of rDNA repeat in Sup mutants, we performed pulsed field gel electrophoresis and subsequent Southern blotting using 5S rRNA gene as a probe (Fig. 3a). As with the previous report, Sup mutant showed retarded migration of chromosome XII compared with SH8836 strain, suggesting that expansion of rDNA cluster had occurred. To determine the precise rDNA repeat copy number, quantitative realtime PCR was performed by comparison with ACT1 gene as a representative of one copy gene (Fig. 3c). The copy number of rDNA repeats in SH8836 strain was approximately 100, while that in Sup mutants varied between 400 and 700. From these results, we concluded that rDNA expansion had occurred in all Sup mutants, and it was suggested that polymerase switch also had occurred in the Sup mutants. In the next step, we measured the copy number of rDNA repeats in Sup mutants harboring pRRN5 plasmid. To this end, we performed PFGE and Southern blotting using 5S rRNA gene as a probe (Fig. 3b) and quantitative real-time PCR (Fig. 3c). The results indicated that rDNA repeat was contracted to almost the same level as wild type (ca. 150-200) and thus, it was suggested that polymerase switching was also abolished by the reintroduction of RRN5 gene. We speculated that the contraction of rDNA repeats might be the reason for not increasing of total RNA level in Sup mutants harboring pRRN5.

\section{Combination of suppressor mutation and fob1 deletion causes concerted increase of intracellular RNA content}

In S. cerevisiae, rDNA replication fork barrier site binding protein Fob1 plays an important role in stabilizing rDNA repeat copy number at a defined number (Brewer and Fangman 1988; Kobayashi and Horiuchi 1996; Linskens and Huberman 1988). It was reported that deletion of $F O B 1$ gene maintains rDNA repeat copy number at a constant level. For this reason, we inferred that if FOB1 gene is deleted, it might lead to increased total RNA content by the maintenance of expanded rDNA repeats observed in Sup mutant even after introduction of pRRN5. To validate this idea, we disrupted the FOB1 gene in all Sup mutants. We checked the rDNA repeat copy number in fob1-deleted Sup mutants by quantitative real-time PCR (Fig. 4). rDNA repeat copy number varied between 300 copies and 800 copies. Among the mutants, $\Delta f o b 1 S u p 13$ and $\Delta f o b 1 S u p 16$ showed the highest copy number (ca. 800 copies).

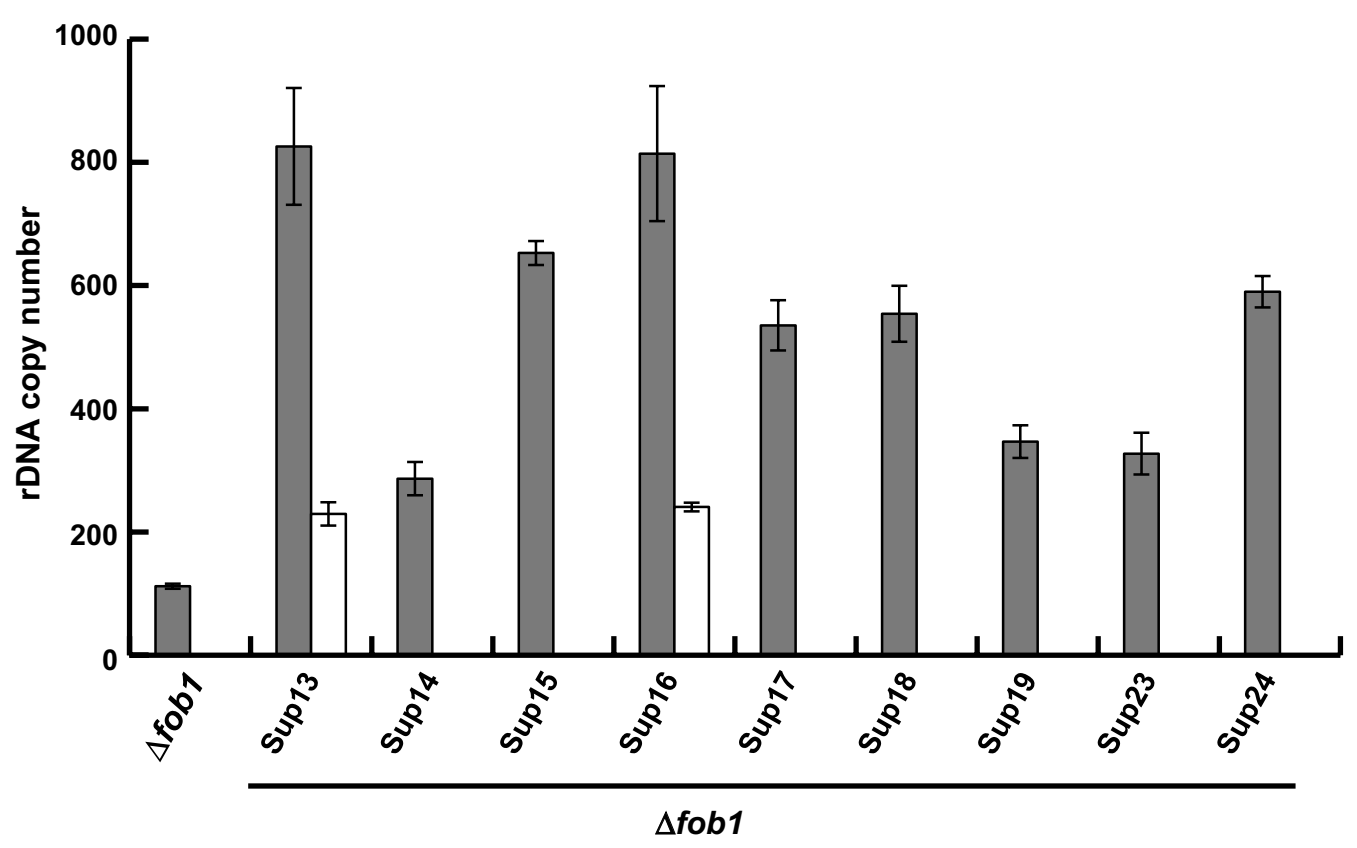

Fig. 4 Determination of rDNA copy number of $\triangle$ fobisup mutants. rDNA copy number in $\triangle$ fob 1 Sup mutants with or without pRRN5 determined by quantitative real-time PCR. Experiments were performed in triplicates. Grey bars $\triangle$ fob 1Sup mutants, white bars $\triangle$ fob1Sup mutants harboring pRRN5 
Then, we introduced pRRN5 plasmid into $\Delta$ fob1Sup13 and $\Delta$ fob1Sup16 and measured the rDNA repeat copy number by quantitative real-time PCR (Fig. 4). Unexpectedly, rDNA repeat copy number was decreased to almost the same level as wild type. The reason of this unexpected result is discussed in the Discussion section. However, we happened to discover that total RNA of $\Delta$ fob1Sup16 harboring pRRN5 ( $\Delta$ fob1Sup16[pRRN5]) were significantly increased $16.5 \%$ compared with wild type even though rDNA copy number was not increased (Fig. 5), whereas, either $\Delta f o b 1$ strain or $\Delta f o b 1 S u p 13$ harboring pRRN5 ( $\Delta$ fob1Sup13[pRRN5]) did not show significant increase of RNA content. We did not measure the RNA content of $\Delta$ fob1Sup13 and $\Delta f o b 1$ Sup 16 , because growth rate of these mutants was considerably much lower than that of other strains. We took this result to suggest that combination of $f o b 1$ gene disruption and SUP16 mutation leads to increase of total RNA content even when functional

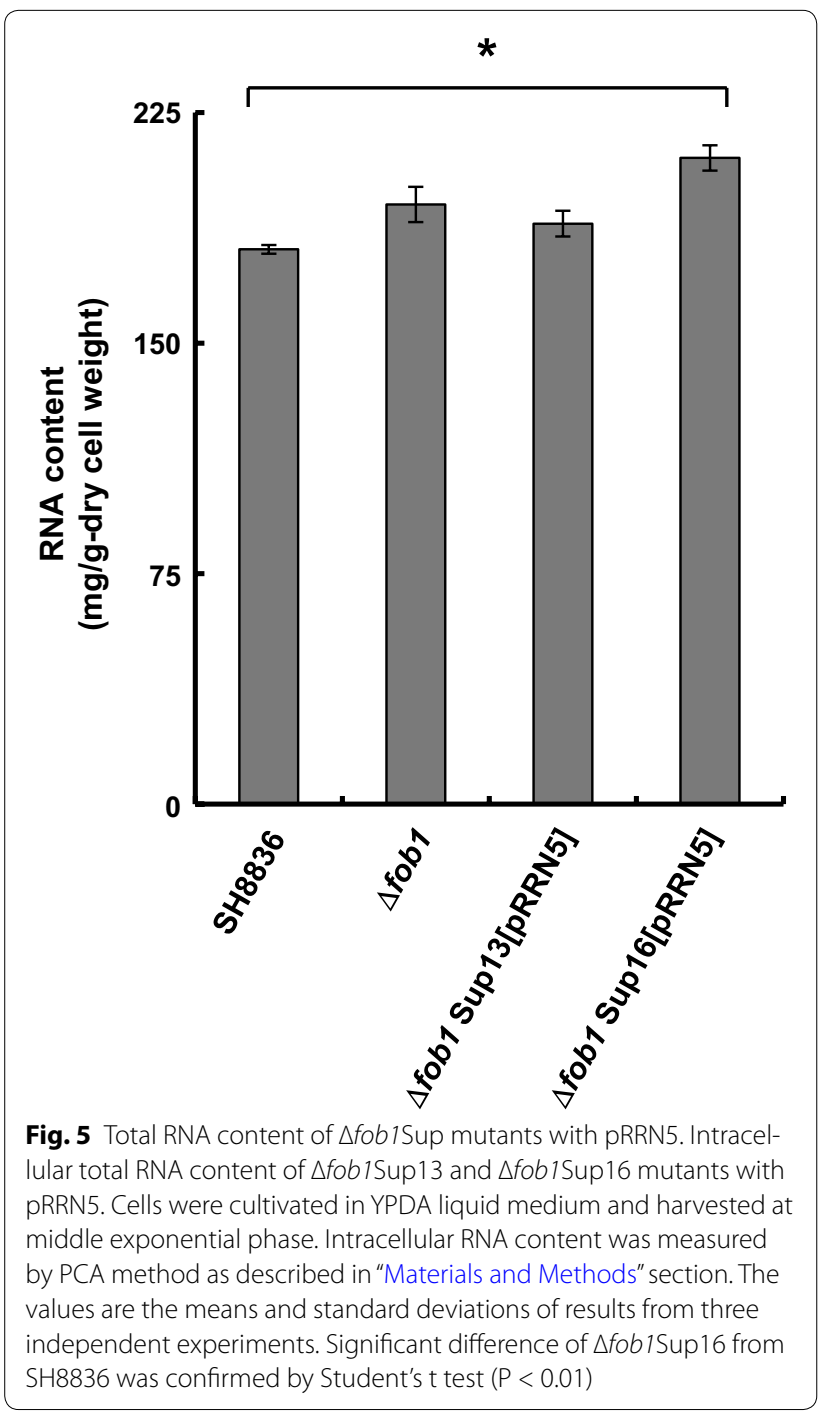

RRN5 gene is reintroduced. This phenotype is stably maintained because the same results were observed after five times subculture (data not shown).

\section{Transcription of NTS2 region is remarkably increased in $\Delta$ fob1Sup 16 strain}

In order to reveal what kind of RNA species affected the increased intracellular RNA content observed in the $\Delta$ fob1Sup16[pRRN5] strain, we performed quantitative real-time PCR using primer pairs distinguishing upper, middle, or lower part of the NTS regions and 18S rRNA (Fig. 6). The result indicated that expression levels of $18 \mathrm{~S}$ rRNA region in all strains tested were almost the same. On the other hand, expression level of NTS1 and NTS2 regions were concertedly increased in $\Delta f o b 1$ Sup16[pRRN5] strain, while both $\Delta f o b 1$ strain and Sup16[pRRN5] showed only slight increase compared with wild type. In particular, expression of the upper part of NTS2 region (NTS2-U) was markedly increased in the $\Delta$ fob1Sup16[pRRN5] strain. It was suggested that the transcript observed at NTS2-U is distinct from that observed at $18 \mathrm{~S}$ rRNA, because almost no expression was observed in the lower part of NTS2 (NTS2-L). We suggest that this increase of NTS2-U is probably attributed to the increased RNA content in the $\Delta f o b 1$ Sup16[pRRN5] strain.

\section{Discussion}

Here, we succeeded in construction of a yeast strain with high RNA content by focusing on Rrn5, an essential rRNA transcription regulator. We isolated nine suppressor mutants of rrn 5 disruption. By combination of $F O B 1$ gene deletion and suppressor mutations, we were able to construct a novel yeast strain showing increased intracellular RNA content.

It was reported that copy number of rDNA repeat is invariable in fob1 deletion (Defossez et al. 1999; Johzuka and Horiuchi 2002; Kobayashi et al. 1998). However, rDNA copy number was altered to almost the same level as wild type in all $\Delta$ fob1Sup mutants with pRRN5 plasmid in this study. The reason of this discrepancy might be explained by one or more following reasons. (i) It was reported that when non-coding RNA transcription in NTS1 region driven by Pol II dependent E-PRO promoter is active, cohesins at the NTS region was removed and a recombination called unequal sister-chromatid recombination is induced, resulting in changes in rDNA copy number (Kobayashi 2011; Kobayashi and Ganley 2005). In $\Delta$ fob1Sup[pRRN5] strain, substantial transcription was observed in NTS1 region as well as NTS2 region (Fig. 6), suggesting that recombination is induced in $\Delta$ fob1Sup[pRRN5] strain. (ii) Abnormally high copy number of rDNA repeat makes it unstable, thus it is easily popped out to yield extra-chromosomal rDNA 


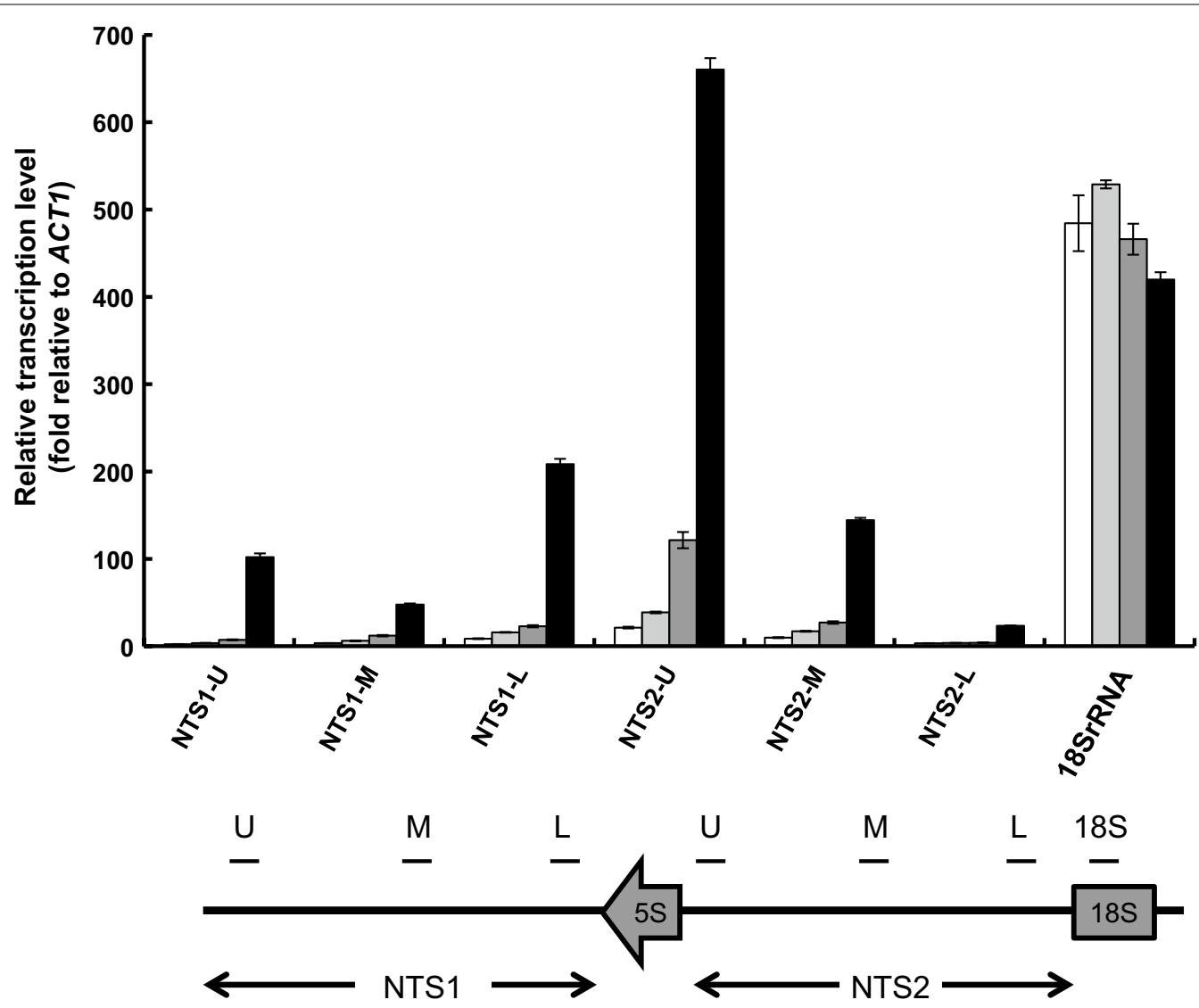

Fig. 6 Transcription level of NTS regions. Transcription level of seven sites within rDNA unit was determined by quantitative real-time PCR. Primer pairs used for detection of each site are listed in Additional file 1: Table S1. White bars; SH8836. Light grey bars Sup 16[pRRN5], dark grey bars $\Delta f \circ b 1$; Black bars $\triangle$ fob1Sup16[pRRN5]. The values are the means and standard deviations of results from three independent experiments. The figure below the graph represents the location of each site in this experiment

circles (ERCs) by Fob1-independent manner. ERC is accumulated only in the mother cell, and its accumulation induces senescence (Kobayashi 2011). Eventually, the number of rDNA copy number becomes closer to that of wild type level.

Within the NTS regions, upstream region of NTS2 (NTS2-U) showed the highest transcription, even higher than $18 \mathrm{~S}$ rRNA region. This drastically high transcription accounts for the increased intracellular RNA content observed in $\Delta$ fob1Sup16[pRRN5] strain. In the previous report, Pol II-driven promoter (C-PRO) is located at the NTS2 region (Cesarini et al. 2010; Li et al. 2006). However, it seems unlikely that C-PRO is responsible for increased transcript observed at NTS2-U, because the precise location of C-PRO is at lower part of the NTS2 (corresponding to NTS2-L). Instead, Li et al. reported that an unidentified transcription with the direction from NTS2-U to NTS2-L is observed in the absence of Sir2 (Li et al. 2006). The average size of this cryptic transcript is approximately $1.0 \mathrm{~kb}$, which is comparable to $15 \%$ of $35 \mathrm{~S}$
rRNA transcript $(6.9 \mathrm{~kb})$. Therefore, this transcript might be responsible for the increased transcription observed at NTS2-U in $\Delta$ fob1Sup16[pRRN5] strain because Sir2 recruitment is regulated by Fob1 (Huang and Moazed 2003). It is likely that abundance of Sir2 is reduced in the absence of Fob1 as supported by the observation that the transcription at NTS2-U is slightly increased in $\Delta$ fob1 strain. Thus, it is reasonable that the transcription at NTS2-U is highly induced in $\Delta$ fob1Sup16[pRRN5] strain by the combination of fob1 deletion, Sup16 mutation, and RRN5 reintroduction.

In this study, we did not identify SUP16 suppressor gene yet. SUP16 suppressor gene was shown to be dominant from dominant/recessive test by crossing Sup16 strain and $\Delta r r n 5$ strain with a helper plasmid (data not shown) and Sup16[pRRN5] strain showed slight NTS transcription like $\Delta f o b 1$ strain. From these results, SUP16 mutation might be a mutation concerning NTS silencing like Sir2 or other RENT complex factors (Huang and Moazed 2003). Chromatin remodeling factor is also a candidate 
for SUP16 because chromatin remodeling is concomitant with NTS transcription. Although SUP16 suppressor gene is not identified at present, we suggest that it synergistically induces NTS transcription with fob1 deletion by unknown mechanism even when Rrn5 is functional, and thereby leading to increased intracellular RNA content. Further studies, especially those including identification of the SUP16 gene are needed to clarify the mechanism.

Previously, we constructed a S. cerevisiae strain accumulating approximately $120 \mathrm{mg} / \mathrm{g}$-Dry cell weight RNA using suppressor mutants obtained from rrn10 disruptant (Chuwattanakul et al. 2011). In this study, we were successful for constructing a strain accumulating approximately $200 \mathrm{mg} / \mathrm{g}$-Dry cell weight RNA by exploiting Rrn5. To our knowledge, this value is higher than any other RNA accumulating yeasts reported so far, although it may not be possible to precisely compare those productivities because culture conditions and medium are different in each report. We propose a novel breeding strategy exploiting transcription of NTS region for high RNA content yeast. Our mutants isolated in this study will be useful not only for an industrial application but for the elucidation of transcriptional regulation of rDNA.

\section{Additional file}

Additional file 1. Addiitonal figures and tables.

\section{Abbreviations}

NTS: non transcribed spacer; UAF: upstream activating factor; CF: core factor; RFB: replication fork barrier; 5-FOA: 5-fluoroorotic acid; PCA: perchloric acid; PFGE: pulsed field gel electrophoresis.

\section{Authors' contributions}

YS designed and performed the experiments, and wrote the manuscript. TK and SU performed the experiments. MS and SH designed and supervised the study, and wrote the manuscript. All authors read and approved the final manuscript.

\section{Author details}

1 Department of Biotechnology, Graduate School of Engineering, Osaka University, 2-1 Yamadaoka, Suita-shi, Osaka 565-0871, Japan. ${ }^{2}$ Department of Applied Microbial Technology, Faculty of Biotechnology and Life Science, Sojo University, Ikeda 4-22-1, Kumamoto-shi, Kumamoto 860-0082, Japan.

\section{Acknowledgements}

We thank Dr. Takehiko Kobayashi (University of Tokyo) for kindly providing the p2453 plasmid. We thank the National Bio-Resource Project (NBRP), Japan, by which SH6471 strain was provided.

\section{Competing interests}

The authors declare that they have no competing interests.

\section{Availability of data and materials}

The datasets supporting the conclusions of this article are included within the article and its Additional file 1.

\section{Ethical approval}

This article does not contain any studies with human participants or animals performed by any of the authors.

\section{Funding}

This work was partially supported by Grant-in-Aid for Challenging Exploratory Research [24658081] (to S. H.) from the Ministry of Education, Culture, Sports, Science and Technology (MEXT) of Japan.

Received: 3 January 2017 Accepted: 20 January 2017

Published online: 02 February 2017

\section{References}

Barbalat R, Ewald SE, Mouchess ML, Barton GM (2011) Nucleic acid recognition by the innate immune system. Annu Rev Immunol 29:185-214. doi:10.1146/annurev-immunol-031210-101340

Brewer BJ, Fangman WL (1988) A replication fork barrier at the $3^{\prime}$ end of yeast ribosomal RNA genes. Cell 55(4):637-643

Cesarini E, Mariotti FR, Cioci F, Camilloni G (2010) RNA polymerase I transcription silences noncoding RNAs at the ribosomal DNA locus in Saccharomyces cerevisiae. Eukaryot Cell 9(2):325-335. doi:10.1128/ec.00280-09

Chuwattanakul V, Kim YH, Sugiyama M, Nishiuchi H, Miwa H, Kaneko Y, Harashima S (2011) Construction of a Saccharomyces cerevisiae strain with a high level of RNA. J Biosci Bioeng 112(1):1-7. doi:10.1016/j. jbiosc.2011.03.011

Chuwattanakul V, Sugiyama M, Khatun F, Kurata K, Tomita I, Kaneko Y, Harashima S (2012) Increased transcription of NOP15, involved in ribosome biogenesis in Saccharomyces cerevisiae, enhances the production yield of RNA as a source of nucleotide seasoning. J Biosci Bioeng 114(1):17-22. doi:10.1016/j.jbiosc.2012.02.022

Defossez PA, Prusty R, Kaeberlein M, Lin SJ, Ferrigno P, Silver PA, Keil RL, Guarente L (1999) Elimination of replication block protein Fob1 extends the life span of yeast mother cells. Mol Cell 3(4):447-455

Goetze H, Wittner M, Hamperl S, Hondele M, Merz K, Stoeckl U, Griesenbeck J (2010) Alternative chromatin structures of the 35S rRNA genes in Saccharomyces cerevisiae provide a molecular basis for the selective recruitment of RNA polymerases I and II. Mol Cell Biol 30(8):2028-2045. doi:10.1128/ mcb.01512-09

Huang J, Moazed D (2003) Association of the RENT complex with nontranscribed and coding regions of rDNA and a regional requirement for the replication fork block protein Fob1 in rDNA silencing. Genes Dev 17(17):2162-2176. doi:10.1101/gad.1108403

Johzuka K, Horiuchi T (2002) Replication fork block protein, Fob1, acts as an rDNA region specific recombinator in S. cerevisiae. Genes Cells 7(2):99-113

Keener J, Josaitis CA, Dodd JA, Nomura M (1998) Reconstitution of yeast RNA polymerase I transcription in vitro from purified components. TATA-binding protein is not required for basal transcription. J Biol Chem 273(50):33795-33802

Keys DA, Vu L, Steffan JS, Dodd JA, Yamamoto RT, Nogi Y, Nomura M (1994) RRN6 and RRN7 encode subunits of a multiprotein complex essential for the initiation of rDNA transcription by RNA polymerase I in Saccharomyces cerevisiae. Genes Dev 8(19):2349-2362

Keys DA, Lee BS, Dodd JA, Nguyen TT, Vu L, Fantino E, Burson LM, Nogi Y, Nomura M (1996) Multiprotein transcription factor UAF interacts with the upstream element of the yeast RNA polymerase I promoter and forms a stable preinitiation complex. Genes Dev 10(7):887-903

Khatun F, Kurata K, Chuwattanakul V, Sugiyama M, Kaneko Y, Harashima S (2013a) Increased transcription of RPL $40 A$ and RPL $40 B$ is important for the improvement of RNA production in Saccharomyces cerevisiae. J Biosci Bioeng 116(4):423-432. doi:10.1016/j.jbiosc.2013.04.006

Khatun F, Sasano Y, Sugiyama M, Kaneko Y, Harashima S (2013b) Increase in rRNA content in a Saccharomyces cerevisiae suppressor strain from rrn10 disruptant by rDNA cluster duplication. Appl Microbiol Biotechnol 97(20):9011-9019. doi:10.1007/s00253-013-5065-9

Kobayashi T (2011) Regulation of ribosomal RNA gene copy number and its role in modulating genome integrity and evolutionary adaptability in yeast. Cell Mol Life Sci 68(8):1395-1403. doi:10.1007/s00018-010-0613-2

Kobayashi T, Ganley AR (2005) Recombination regulation by transcriptioninduced cohesin dissociation in rDNA repeats. Science 309(5740):15811584. doi:10.1126/science. 1116102 
Kobayashi T, Horiuchi T (1996) A yeast gene product, Fob1 protein, required for both replication fork blocking and recombinational hotspot activities. Genes Cells 1(5):465-474

Kobayashi T, Heck DJ, Nomura M, Horiuchi T (1998) Expansion and contraction of ribosomal DNA repeats in Saccharomyces cerevisiae: requirement of replication fork blocking (Fob1) protein and the role of RNA polymerase I. Genes Dev 12(24):3821-3830

Kurihara K, Kashiwayanagi M (2000) Physiological studies on umami taste. J Nutr 130(4S Suppl):931S-934S

Lalo D, Steffan JS, Dodd JA, Nomura M (1996) RRN11 encodes the third subunit of the complex containing Rrn6p and Rrn7p that is essential for the initiation of rDNA transcription by yeast RNA polymerase I. J Biol Chem 271(35):21062-21067

Li C, Mueller JE, Bryk M (2006) Sir2 represses endogenous polymerase II transcription units in the ribosomal DNA nontranscribed spacer. Mol Biol Cell 17(9):3848-3859. doi:10.1091/mbc.E06-03-0205

Lin CW, Moorefield B, Payne J, Aprikian P, Mitomo K, Reeder RH (1996) A novel 66-kilodalton protein complexes with Rrn6, Rrn7, and TATA-binding protein to promote polymerase I transcription initiation in Saccharomyces cerevisiae. Mol Cell Biol 16(11):6436-6443

Linskens MH, Huberman JA (1988) Organization of replication of ribosomal DNA in Saccharomyces cerevisiae. Mol Cell Biol 8(11):4927-4935

Oakes M, Siddiqi I, Vu L, Aris J, Nomura M (1999) Transcription factor UAF, expansion and contraction of ribosomal DNA ( $r D N A$ ) repeats, and RNA polymerase switch in transcription of yeast rDNA. Mol Cell Biol 19(12):8559-8569

Petes TD (1979) Yeast ribosomal DNA genes are located on chromosome XII. Proc Natl Acad Sci USA 76(1):410-414
Rathbone MP, Christjanson L, Deforge S, Deluca B, Gysbers JW, Hindley S, Jovetich M, Middlemiss P, Takhal S (1992) Extracellular purine nucleosides stimulate cell division and morphogenesis: pathological and physiological implications. Med Hypotheses 37(4):232-240

Sheehan C, Weiss AS (1990) Yeast artificial chromosomes: rapid extraction for high resolution analysis. Nucleic Acids Res 18(8):2193

Sikorski RS, Hieter P (1989) A system of shuttle vectors and yeast host strains designed for efficient manipulation of DNA in Saccharomyces cerevisiae. Genetics 122(1):19-27

Steffan JS, Keys DA, Dodd JA, Nomura M (1996) The role of TBP in rDNA transcription by RNA polymerase I in Saccharomyces cerevisiae: TBP is required for upstream activation factor-dependent recruitment of core factor. Genes Dev 10(20):2551-2563

Sugiyama M, Ikushima S, Nakazawa T, Kaneko Y, Harashima S (2005) PCRmediated repeated chromosome splitting in Saccharomyces cerevisiae. Biotechniques 38(6):909-914

Vu L, Siddiqi I, Lee BS, Josaitis CA, Nomura M (1999) RNA polymerase switch in transcription of yeast rDNA: role of transcription factor UAF (upstream activation factor) in silencing rDNA transcription by RNA polymerase II. Proc Natl Acad Sci USA 96(8):4390-4395

Warner JR (1999) The economics of ribosome biosynthesis in yeast. Trends Biochem Sci 24(11):437-440

Yamamoto RT, Nogi Y, Dodd JA, Nomura M (1996) RRN3 gene of Saccharomyces cerevisiae encodes an essential RNA polymerase I transcription factor which interacts with the polymerase independently of DNA template. The EMBO J 15(15):3964-3973

\section{Submit your manuscript to a SpringerOpen ${ }^{\odot}$ journal and benefit from:}

- Convenient online submission

- Rigorous peer review

- Immediate publication on acceptance

- Open access: articles freely available online

- High visibility within the field

- Retaining the copyright to your article

Submit your next manuscript at springeropen.com 